\title{
Prediction of Fault-proneness at Early Phase in Object-Oriented Development
}

\author{
Toshihiro Kamiya, Shinji Kusumoto and Katsuro Inoue \\ Graduate School of Engineering Science, Osaka University \\ 1-3 Machikaneyama, Toyonaka, Osaka, 560-8531, Japan \\ E-mail: kamiya@ics.es.osaka-u.ac.jp
}

\begin{abstract}
To analyze the complexity of object-oriented software, several metrics have been proposed. Among them, Chidamber and Kemerer's metrics are well-known ones as object-oriented metrics. Also, the effectiveness has been empirically evaluated from the viewpoints of estimating the fault-proneness of object-oriented software. In the evaluations, their metrics were applied to not design specification but the source code because some of them measure an inner complexity of a class, and such information can not be obtained until the algorithm and structure of the class are determined at the end of design phase. However, the estimation of the fault-proneness should be done in the early phase to effectively allocate effort for fixing the faults. This paper proposes a new method to estimate the fault-proneness of the class in the early phase, using several complexity metrics for object-oriented software. In the proposed method, we introduce four checkpoints into the analysis / design / implementation phase, and estimate the fault-prone classes using the applicable metrics at each checkpoint.
\end{abstract}

\section{Introduction}

In attempt to reduce the number of delivered faults, it was reported that most companies spend between 50-80\% of their software development effort on testing[7]. Therefore, reducing the effort of testing is a key to high productivity in software development. Software review is one of the most effective techniques to reduce the testing effort. In order to effectively review and test the software products, it is needed to identify the fault-prone modules so that review and testing effort can be concentrated on the modules[1]. Several complexity metrics have already been proposed to localize such the fault-prone modules. Then,
Chidamber and Kemerer proposed six complexity metrics for OO software [5]. However, the metrics suite was applied to the source code because some of their metrics measure an inner complexity of a class, and such information cannot be obtained until the algorithm and structure of the class are determined at the end of design phase. The estimation of the fault-proneness should be done in the early phase to effectively allocate the effort for fixing the faults.

This paper proposes a new method to estimate the fault-proneness of the class in the early design phase, using several complexity metrics for OO software. In the proposed method, we introduce four checkpoints into the development process based on OMT[14]. According to the data obtained from the products (design specification and source code) at each checkpoint, we use only the applicable metrics among the complexity metrics and estimate the fault-proneness by using the multivariate logistic regression analysis[18]. Then, we have applied the proposed method to an experimental project held at a computer company. The analysis result of the experiment shows the validity and usefulness of the proposed method in the estimation of the fault-proneness.

\section{Preliminary}

\subsection{Object-Oriented Design Method}

There exist several object-oriented design methods [2][6][14][15]. Among them, OMT[14] is a broad methodology that addresses most aspects of the $\mathrm{OO}$ analysis and design technology and incorporates the lessons learned from real projects as well as from other methodologies.

OMT consists of three phases: Analysis, System Design and Object Design. Analysis is concerned with under- 
standing and modeling the application and the domain within which it operates. System Design determines the overall architecture of the system. The system is organized into subsystems. Concurrency is organized by grouping objects into concurrent tasks. Object Design elaborates, refines and then optimizes the analysis models to produce a practical design. Algorithms and data structures used in the classes are determined.

From the viewpoint of the structure of the class, elaboration is carried out as the following six steps:

1. Identify classes in the target system.

2. Identify the reference-relationships between the classes.

3. Identify the attributes of the classes.

4. Determine the derivation-relationships between the classes.

5. Define the operations based on the functional models.

6. Design the algorithms to implement the operations.

\subsection{Complexity Metrics for Object-Oriented Software}

Software metrics are quantitative measures of software products and process[8]. Especially, the complexity metric for a program code is the most well-known metric, and is usually used in the implementation phase and test phase, since it is a good indicator of whether the product is welldesigned, understandable, and easy to modify. For example, Software Science by Halstead[9] and Cyclomatic Number by McCabe have been used in many software development organizations. Then, Chidamber and Kemerer[5] have defined a suite of metrics for OO design. The metrics suite includes six metrics and mainly evaluate the relationship between the classes on the design specification. Each metric evaluates the complexity of the target class and the definitions of the metrics are as follows[5]:

\section{WMC(weighted methods per class):}

Consider the target class $C_{1}$, with methods $M_{1}, \ldots, M_{n}$, that are related in the class. Let $c_{1}, \ldots, c_{n}$ be the complexity of the methods. Then $W M C=\Sigma c_{i}$. You ought to choose an adequate interval scale metric $f$ that gives $c_{i}=$ $f\left(M_{i}\right)$.

In both [1] and [5], they made an assumption that all method complexities are considered to be unity, then WMC is the number of methods. We also use this assumption, so we plainly employ a term NIM(Number of Instance Methods)[12] as WMC.

\section{DIT(depth of inheritance tree):}

Depth of inheritance of the target class is the DIT metric for the class.

\section{NOC(number of children):}

NOC means the number of immediate subclass subordinated to the target class in the class hierarchy.

\section{CBO(coupling between object classes):}

CBO for the target class is a count of the number of other classes to which it is coupled. A class is coupled to another if it uses is methods or instance variables of another.

\section{RFC(response for a class):}

$R F C=|R S|$ where $R S$ is the response set for the class. $R S$ $=M \cup\left(\cup_{\text {all } i} R_{i}\right)$ where $R_{i}=$ set of methods called by method $i$ and $M=$ set of all methods in the class.

\section{LCOM(lack of cohesion in methods):}

Consider the target class $C_{1}$ with $\$ \mathrm{n} \$$ methods $M_{1}, \ldots, M_{n}$. Let $I_{i}=$ set of instance variables used by methods $M_{i}$. There are $n$ such sets $I_{1}, \ldots, I_{\mathrm{n}}$. Let $P=\left\{\left(I_{i}, I_{j}\right) \mid I_{i} \cap l_{j}=\right.$ $\phi\}$ and $Q=\left\{\left(I_{i}, I_{j}\right) \mid I_{i} \cap I_{j} \neq \phi\right\}$. If all $n$ sets $I_{l}, \ldots, I_{n}$ are $\phi$ then let $P=\phi . L C O M=\max (|P|-|Q|, 0)$.

Besides the above metrics, NIV (Number of Instance Variables) is frequently used as a design metric of objectoriented software[12].

\section{NIV(number of instance variables):}

NIV is a number of instance variables of the target class.

\subsection{Evaluation of Complexity Metrics}

Chidamber and Kemerer have presented empirical data on their metrics from actual commercial systems. They have adopted the Weyuker's properties to evaluate their metrics (Weyuker has developed a list of desiderata for software metrics and has evaluated a number of existing software metrics using these properties[16]), and concluded that the metrics are generally satisfied the Weyuker's properties. Basili et al. empirically evaluated that Chidamber and Kemerer's OO metrics show to be better predictors than the best set of traditional code metrics[1]. 
Table 1. Checkpoint and available metrics.

\begin{tabular}{l|l|l}
\hline Checkpoint & Added Information & Available Metrics \\
\hline \hline $\begin{array}{l}\text { (CP1) } \\
\text { Entity and Relation }\end{array}$ & $\begin{array}{l}\text { Reference-relationship among classes and } \\
\text { attributes of classes }\end{array}$ & NIV, CBON \\
\hline $\begin{array}{l}\text { (CP2) } \\
\text { Structure and inheritance }\end{array}$ & Class hierarchy, methods and reused library & $\begin{array}{l}\text { NIV, CBON, CBOR, CBO, NIM, DIT, } \\
\text { NOC }\end{array}$ \\
\hline $\begin{array}{l}\text { (CP3) } \\
\text { Algorithm }\end{array}$ & Algorithm of the methods & $\begin{array}{l}\text { NIV, CBON, CBOR, CBO, NIM, DIT, } \\
\text { NOC, RFC, LCOM }\end{array}$ \\
\hline $\begin{array}{l}\text { (CP4) } \\
\text { Implementation }\end{array}$ & Source code & $\begin{array}{l}\text { NIV, CBON, CBOR, CBO, NIM, DIT, } \\
\text { NOC, RFC, LCOM, SLOC }\end{array}$ \\
\hline
\end{tabular}

In both [1] and [5], they collected the metrics values from source codes. But the design specification is constructed in the earlier development phase. Thus, by applying the metrics to the design specification, it is possible to estimate the fault proneness more effectively. It would produce efficient fault detection to do the resourceallocation and coordinate the scheduling based on the result of fault-estimation. However, it is very difficult to apply the metrics to the design specification. For example, with respect to calculating the RFC and LCOM, we need the detail information about the algorithms in the method and call-relationship between the methods. These informations are usually described on the design specification at the later design phase (just before the implementation phase).

\section{Proposed Method}

\subsection{Key Idea}

We regard the analysis / design / implementation phase as a series process in which the information about software product gradually increases as the process progresses. As described in Section 2, some of the metrics can be applied to the design specification at the earlier design phase, and some of them can be applied at the later design phase. Based on the fact, as design phase progress, we estimate the fault-proneness only using the applicable metrics to the design specification.

At first, we introduce four checkpoints in the development process and identify which information has been added to the design specification at each checkpoint. Then, we define the subset of the conventional metrics applicable to the design specification developed at each checkpoint. Finally, at each checkpoint, we estimate the fault- proneness of the class using the multivariate logistic regression analysis with the applicable metrics.

\subsection{Checkpoints and Applicable Metrics}

In Section 2, we divided the development process of OMT into the following six steps. Based on the division, we introduce the four checkpoints in the analysis / design / implementation phase of OMT.

\section{(CP1) Entity and relation}

CP1 is the checkpoint where Steps 1, 2 and 3 have been completed. That is, reference-relationship between classes (coupling) and attributes (instance variable) of the classes have been determined. NIV can be calculated from the attribute information. The derivation-relationships have not been described. $\mathrm{CBO}$ can be calculated from the reference-relationship between classes, but reference to the reused class is not clearly described so that the value of $\mathrm{CBO}$ is not correct.

\section{(CP2) Structure and inheritance}

CP2 is the checkpoint where Steps 4 and 5 have been completed. That is, derivation between classes and the methods in the classes have been determined and class hierarchy tree is clearly described. Thus, DIT can be calculated. NIM can be calculated from the information of the methods. The reused classes are determined so that $\mathrm{CBO}$ can be calculated correctly.

\section{(CP3) Algorithm}

CP3 is the checkpoint where Step 6 has been completed. That is, algorithms in each method and call-relationship between the methods are determined. Based on the information, LCOM and RFC can be calculated. 


\section{(CP4) Implementation}

CP4 is the checkpoint where source code has been implemented. For each class, SLOC (source lines of code) can be calculated.

$\mathrm{CBO}$ at $\mathrm{CP} 1$ (hereafter: $\mathrm{CBON}, \mathrm{CBO}$ newly developed) differs from original definition. However, our previous research result showed that $\mathrm{CBON}$ have highly correlated with the fault-proneness[10]. We also introduce $C B O R$ (CBO reused) that is defined as $C B O R=C B O-$ $C B O N$. Table 1 summarizes the checkpoints and the metrics which can be calculated at each checkpoint.

\subsection{Multivariate Logistic Regression Analysis}

Recently, the multivariate logistic regression analysis, whose inputs are several metrics, has been frequently used to evaluate the fault-proneness of the program[1][3].

A multivariate logistic regression model is based on following relationship equation:

$$
P\left(X_{1}, \ldots, X_{n}\right)=1 /\left\{1+\exp \left(-\left(C_{0}+C_{1} X_{1}+\ldots+C_{\mathrm{n}} X_{\mathrm{n}}\right)\right)\right\}
$$

where $P$ is the probability that errors is found in a class, and $X_{\mathrm{i}} \mathrm{s}$ are metrics of the class. If given metrics values make $P$ greater than 0.5 , the class is predicted to have faults (fault prone).

To obtain particular prediction equation $P\left(X_{1}, \ldots, X_{n}\right)$, we need to determine the coefficients $C_{0}, \ldots, C_{n}$ by step-

Table 2. Descriptive statistics of the $141 \mathrm{C}++$ classes.

\begin{tabular}{l|r|r|r|r|r}
\hline Metrics & Min. & Max. & $\begin{array}{l}\text { Me- } \\
\text { dian }\end{array}$ & Mean & Std. Dev. \\
\hline \hline NIV & 0 & 14 & 3 & 4.00 & 2.67 \\
\hline CBO & 0 & 5 & 1 & 1.39 & 1.59 \\
\hline CBON & 0 & 3 & 0 & 0.53 & 0.99 \\
\hline CBOR & 0 & 4 & 1 & 0.86 & 0.99 \\
\hline NIM & 0 & 22 & 3 & 5.73 & 4.86 \\
\hline DIT & 0 & 6 & 4 & 3.44 & 1.41 \\
\hline NOC & 0 & 0 & 0 & 0.00 & 0.00 \\
\hline RFC & 0 & 27 & 7 & 8.23 & 6.81 \\
\hline LCOM & 0 & 190 & 3 & 22.42 & 36.84 \\
\hline SLOC & 0 & 420 & 71 & 96.43 & 81.01 \\
\hline Ec & 0 & 17 & 0 & 0.57 & 1.93 \\
\hline Et(min.) & 0 & 599 & 0 & 12.68 & 58.94 \\
\hline \multicolumn{1}{|c|}{ Ec is } & & & & &
\end{tabular}

$E c$ is the number of faults detected in the class.

$E t$ is the time spent for fixing the faults. wise variable selection process[18], using observed metrics and fault data.

\section{Empirical Evaluation}

\subsection{Outline of the experiment}

The experimental project was performed at a computer company for five days in August 1997. The main characteristics of the project can be summarized as follows:

(1) The Developers were new employees of the computer company and had just graduated from college in March 1997. All the developers studied object-oriented design and programming in $\mathrm{C}++$ Language.

(2) There are sixteen developer teams of four or five developers. Each team built a mail deliver system of an identical requirement. The predetermined system requirements, the division to subsystems and subsystem's interface designs were handed over to each team.

(3) After a team declared that its program was finished, the instructors executed an acceptance test.

\subsection{Empirical Data}

We collected complexity metrics and fault data from each developer. Unfortunately, we could not collect the design specification based on OMT in this experiment. We assume that all the information of the design specification

Table 3. Coefficients at Each Checkpoint.

\begin{tabular}{l|c|c|c|c}
\hline \multirow{2}{*}{ Metrics } & \multicolumn{4}{|c|}{ Coefficients } \\
\cline { 2 - 5 } & CP1 & CP2 & CP3 & CP4 \\
\hline \hline C $_{0}$ & -3.37 & -1.23 & -1.31 & -2.69 \\
\hline NIV & 0.420 & EL & EL & EL \\
\hline CBON & EL & EL & EL & EL \\
\hline CBOR & - & 0.934 & 0.890 & EL \\
\hline CBO & - & EL & EL & EL \\
\hline NIM & - & 0.336 & EL & EL \\
\hline DIT & - & -1.16 & -1.28 & -0.663 \\
\hline NOC & - & - & EL & EL \\
\hline RFC & - & - & 0.284 & EL \\
\hline LCOM & - & - & - & EL \\
\hline SLOC & - & - & - & 0.0302 \\
\hline
\end{tabular}

The ' $E L$ ' means that the metric was eliminated form the prediction equation by a backward variable elimination process. The '-'s are not applicable at the checkpoint. 
Table 4. Fault Prediction at CP1.

\begin{tabular}{l|l|c|c}
\hline \multicolumn{2}{l|}{ Prediction } & No fault & \multicolumn{1}{l}{ Fault } \\
\hline \hline Actual & No fault & 112 & 2 \\
\cline { 2 - 4 } & Fault & $18(43)$ & $9(37)$ \\
\hline
\end{tabular}

The figures before parentheses are the number of classes. The figures within parentheses are the number of faults in those classes.

Table 5. Fault Prediction at CP2.

\begin{tabular}{l|l|c|c}
\hline \multicolumn{2}{l|}{ Prediction } & No fault & Fault \\
\hline \hline \multirow{2}{*}{ Actual } & No fault & 109 & 5 \\
\cline { 2 - 4 } & Fault & $11(20)$ & $16(60)$ \\
\hline
\end{tabular}

Table 6. Fault Prediction at CP3.

\begin{tabular}{l|c|c|c}
\hline \multicolumn{2}{l|}{ Prediction } & No fault & \multicolumn{1}{l}{ Fault } \\
\hline \hline \multirow{2}{*}{ Actual } & No fault & 111 & 3 \\
\cline { 2 - 4 } & Fault & $9(18)$ & $18(62)$ \\
\hline
\end{tabular}

Table 7. Fault Prediction at CP4.

\begin{tabular}{l|l|c|c}
\hline \multicolumn{2}{l|}{ Prediction } & No fault & Fault \\
\hline \hline \multirow{2}{*}{ Actual } & No fault & 111 & 3 \\
\cline { 2 - 4 } & Fault & $8(14)$ & $19(66)$ \\
\hline
\end{tabular}

were included in the source code since it was implemented based on the design specification. So, we collected the metrics values, which can be calculated at each checkpoint, from source code by using a metrics tool that extracts nine metrics from $\mathrm{C}++$ source code[11].

We prepared a documentation tool to collect fault data. We have eliminated the data of developers who did not report data or irresponsible data. As a result, seventeen members' data (141 classes and 80 faults) are available. Table 2 shows common descriptive statistics of the metrics distributions.

\subsection{Analysis}

Table 3 shows the prediction model's coefficients calculated by multivariate logistic regression analysis. DIT appeared as a negative factor to complexity. This can be explained by the fact that there were a lot of 'dialog' classes developed and the functionalities were very simple but the their DIT values were relatively large. LCOM is eliminated from a equation at $\mathrm{CP} 4$, and this result agrees with the results in [1].

Tables 4, 5, 6 and 7 show the obtained prediction model by the multivariate logistic regression with the data collected at each checkpoint. For example, in Table 4, 112
Table 8. Fault Predict Precision at Checkpoint.

\begin{tabular}{l|r|r|r|r}
\hline Checkpoint & CP1 & CP2 & CP3 & CP4 \\
\hline \hline Correctness(\%) & 82 & 76 & 85 & 86 \\
\hline Completeness(\%) & 33 & 59 & 63 & 70 \\
\hline $\begin{array}{l}\text { Error-based Com- } \\
\text { pleteness (\%) }\end{array}$ & 46 & 75 & 71 & 83 \\
\hline
\end{tabular}

classes are predicted to be no-faulty and actually no-faulty. Eighteen classes are predicted to be no-faulty but actually faulty (include 43 faults).

Here, we introduce the following three criterions to evaluate the result of the prediction:

Correctness $=C_{\mathrm{PF} \mathrm{AF}} /\left(C_{\mathrm{PF} \mathrm{AF}}+C_{\mathrm{PF} \mathrm{AN}}\right)$

Completeness $=C_{\mathrm{PF} \mathrm{AF}} /\left(C_{\mathrm{PF} \mathrm{AF}}+C_{\mathrm{PN} \mathrm{AF}}\right)$

Completeness $_{\text {error based }}=E_{\mathrm{PF} \mathrm{AF}} /\left(E_{\mathrm{PF} \mathrm{AF}}+E_{\mathrm{PN} \mathrm{AF}}\right)$

where $C_{\mathrm{PF} \mathrm{AF}}$ is the number of predicted faulty and actually faulty classes, $C_{\mathrm{PF} \text { AN }}$ is number of predicted faulty but actually no-faulty classes, $C_{\mathrm{PN}} \mathrm{AF}$ is number of predicted no-faulty but actually faulty classes, $E_{x}$ is number of faults found in corresponding $C_{x}$ classes.

Table 8 shows the estimation accuracy of the fault proneness at each checkpoint. According to the progress of the checkpoints, the estimation accuracy is improved. CP4 is the last phase of the development process and the estimation accuracy at CP4 is the upper limit in this experiment.

Completeness at CP1 is relatively low (33\%). On the other hand, correctness is high $(82 \%)$. Thus, the estimation can be used to seed the fault-prone classes. The seeded classes become the candidates that should be reviewed and tested selectively. Also, the location of the seeded classes might be the criterion of the judgment for review. For example, if the seeded classes are concentrated on the important section of the design specification and the section is difficult to test, we should redesign it.

Though the metrics for algorithms of the method cannot be used at CP2, completeness at CP2 is excellent with respect to the upper limit at $\mathrm{CP} 4$. It suggests that it would be possible to estimate the fault-proneness from the design specification in the design phase where the algorithms are not determined, without source code.

The result of estimation at CP3 fell short of our expectations. We consider that the accuracy would be improved by using 'fine-grained' $\mathrm{C}++$ design metrics[3] together at CP3. The accuracy would be improved by cyclomatic number as WMC. 


\section{Conclusion and Future Work}

In this paper, we proposed a new method to estimate the fault-proneness of the class in the early design phase, using several complexity metrics for $\mathrm{OO}$ software. We have introduced four checkpoints into the analysis/design/ implementation phase, in which particular subsets of metrics are applicable. We have applied the proposed method to an experimental project. The analysis result shows the validity and usefulness of the proposed method. Future research includes the following:

(1) Extension of proposed method:

We are going to extend the proposed method to deal with other $\mathrm{OO}$ design specifications and other complexity metrics[4].

(2) Metrics for dynamic complexity:

Chidamber and Kemerer's metrics evaluate the static complexity of the OO software. Several OO design specifications includes dynamic information. It is necessary to evaluate such dynamic complexity.

\section{(3) Metrics tool for design specification:}

Currently, UML (Unified Modeling Language) has been proposed as the standard description language for the OO design specification[17]. We are going to develop the measurement tool for design specification described in UML.

\section{Acknowledgements}

We would like to thank the support of Mr. Yukio Mohri and Mr. Yousuke Takahashi of Nihon Unisys corporation for the experimental projects in Section 4. We also thank Mr. Shuji Takabayashi of Nara Institute of Science and Technology for his assistance in building a metrics tool.

\section{References}

[1] V. R. Basili, L. C. Briand, W. L. Mélo: A validation of object-oriented design metrics as quality indicators, IEEE Trans. on Software Eng., Vol. 20, No. 22, pp. 751-761 (1996).

[2] G. Booch: Object Oriented Analysis and Design with Applications, The Benjamin / Cummings (1994).

[3] L. C. Briand, P. Devanbu, and W. Mélo: An Investi- gation into Coupling Measures for $\mathrm{C}++$, Proc. of the19th Int'l Conference on Software Eng., Boston, USA, pp.412-421 (1997).

[4] L. C. Briand, J. Daly, V. Porter and J. Wüst: Predicting Fault-Prone Classes with Design Measures in Object-Oriented Systems, Proc. of the 9th Int'l Symposium on Software Reliability Eng., Paderborn, Germany, pp.334-343 (1998).

[5] S. R. Chidamber and C. F. Kemerer: A metrics suite for object-oriented design, IEEE Trans. on Software Eng., Vol. 20, No.6, pp.476-493 (1994).

[6] P. Coad and E. Yourdon: Object Oriented Analysis, 2nd ed., Yourdon Press (1991).

[7] J. S. Collofello and S. N. Woodfield: Evaluating the effectiveness of reliability-assurance techniques, Journal of Systems \& Software, Vol.9, No.3, pp.191195 (1989).

[8] N. E. Fenton, and S. L. Pfleeger, Software Metrics, A Rigorous \& Practical Approach. 2nd ed., Thomson, London (1996).

[9] M. H. Halstead: Element of software science, New York, Elsevier North-Holland (1977).

[10] T. Kamiya, S. Kusumoto, K. Inoue and Y. Mohri: Empirical evaluation of reuse sensitiveness of complexity metrics, Information and Software Technology (to appear).

[11] T. Kamiya, S. Takayabashi, S. Kusumoto and K. Inoue: Measurement tool for complexity of $\mathrm{C}++$ program, Objecto-Oriented '98 Symposium, Tokyo, Japan (1998) (in Japanese).

[12] M. Lorenz and J. Kidd: Object-Oriented software metrics, New Jersey, Prentice Hall (1994).

[13] T. J. McCabe: A complexity measure, IEEE Trans. on Software Eng., Vol. SE-2, No.4, pp.308-320 (1976).

[14] J. Rumbaugh, M. Blaha, W. Premerlani, F. Eddy and W. Lorensen: Object Oriented Modeling and Design, Prentice Hall (1991).

[15] S. Schlaer and S. Mellor: Object Oriented System Analysis, Prentice Hall (1988).

[16] E. J. Weyuker: Evaluating software complexity measures, IEEE Trans. on Software Eng., Vol.14, No.9, pp.1357-1365 (1988).

[17] UML Modeling Language, Standard Software Notation. Available at <http://www.rational.com/>.

[18] SPSS Base 8.0 Applications Guide/Professional Statistics, SPSS (1998). 